\title{
Oscillations above sunspots from the temperature minimum to the corona
}

\author{
N. I. Kobanov, A. A. Chelpanov, and D. Y. Kolobov \\ Institute of Solar-Terrestrial Physics of Siberian Branch of Russian Academy of Science, PO Box 291 Lermontov st., 126-a, \\ Irkutsk 664033, Russia \\ e-mail: kobanov@iszf.irk.ru
}

Received 12 October 2012 / Accepted 3 May 2013

\begin{abstract}
Context. An analysis of the oscillations above sunspots was carried out using simultaneous ground-based and Solar Dynamics Observatory (SDO) observations (Si I $10827 \AA$, He I $10830 \AA$, Fe I $6173 \AA$, $1700 \AA$, He II $304 \AA$, Fe Ix $171 \AA$ ).

Aims. Investigation of the spatial distribution of oscillation power in the frequency range $1-8 \mathrm{mHz}$ for the different height levels of the solar atmosphere. Measuring the time lags between the oscillations at the different layers.

Methods. We used frequency filtration of the intensity and Doppler velocity variations with Morlet wavelet to trace the wave propagation from the photosphere to the chromosphere and the corona.

Results. The 15 min oscillations are concentrated near the outer penumbra in the upper photosphere (1700 $)$, forming a ring that expands in the transition zone. These oscillations propagate upward and reach the corona level, where their spatial distribution resembles a fan structure. The spatial distribution of the $5 \mathrm{~min}$ oscillation power looks like a circle-shape structure matching the sunspot umbra border at the photospheric level. The circle expands at the higher levels (He Ir $304 \AA$ and Fe Ix $171 \AA$ ). This indicates that the low-frequency oscillations propagate along the inclined magnetic tubes in the spot. We found that the inclination of the tubes reaches 50-60 degrees in the upper chromosphere and the transition zone.

The main oscillation power in the $5-8 \mathrm{mHz}$ range concentrates within the umbra boundaries at all the levels. The highest frequency oscillations $(8 \mathrm{mHz})$ are located in the peculiar points inside the umbra. These points probably coincide with umbral dots.

We deduced the propagation velocities to be $28 \pm 15 \mathrm{~km} \mathrm{~s}^{-1}, 26 \pm 15 \mathrm{~km} \mathrm{~s}^{-1}$, and $55 \pm 10 \mathrm{~km} \mathrm{~s}^{-1}$ for the Si I $10827 \AA-\mathrm{He}$ I $10830 \AA$, $1700 \AA$ - $-\mathrm{He}_{\text {II }} 304 \AA$ A , and He II $304 \AA$ A-Fe Ix 171 Å height levels, respectively.
\end{abstract}

Key words. Sun: chromosphere - Sun: oscillations - sunspots - Sun: corona

\section{Introduction}

Sunspot oscillations in the lower solar atmosphere have been observed and discussed for many decades (Beckers \& Tallant 1969; Zirin \& Stein 1972; Giovanelli 1972; Balthasar \& Wiehr 1990; Zhugzhda et al. 1985; Lites 1992; Tsiropoula et al. 2000). The problem has turned out to be complicated (Bogdan \& Judge 2006; Thomas \& Weiss 2008). Despite some success in this field, we are still far from a comprehensive understanding of the wave processes in and above sunspots.

Chromospheric waves in sunspots are readily detected in the $\mathrm{H} \alpha$ and $\mathrm{He}_{\mathrm{I}} 10830 \AA$ lines. It was found that different oscillatory modes coexist and the dominant frequency changes from $6-7 \mathrm{mHz}$ in the umbra to $1.5-2 \mathrm{mHz}$ in the outer penumbra. One hypothesis to explain the observed wave behavior is the common source located in subphotospheric layers. The scenario is that the waves originate in the deep layers of the solar atmosphere and propagate upward along the magnetic field lines (Rouppe van der Voort et al. 2003; Kobanov et al. 2006, 2011; Bloomfield et al. 2007). In this case, one expects a positive time delay for the waves observed at the upper and lower levels. Detecting such a delay is a complex task, and the results from different authors are not always consistent with one another (Kobanov et al. 2011; Centeno et al. 2009).

Recent progress in solar physics instrumentation has revived the interest in investigating sunspot oscillations.
Multiwavelength studies of the 3 and 5 min waves are of main concern. Whereas at the photosphere-chromosphere level these waves are detectable in intensity and Doppler velocity signals, in the transition zone and the corona they are recorded in UV intensity.

Aschwanden et al. (1999) and Nakariakov et al. (1999) researched transverse spatial oscillations of coronal loops, whose footpoints were anchored in the photosphere. The oscillations studied by Transition Region And Coronal Explorer (TRACE) in the $171 \AA$ line were triggered by a flare and their mean period was found to be $280 \mathrm{~s}$. De Moortel et al. (2002) suggested a relation between the 3 min oscillations observed in coronal loops above sunspots and the 5 min oscillations in neighboring loops. These authors interpret the $3 \mathrm{~min}$ oscillations as slow magnetoacoustic waves with a propagation velocity of $70-235 \mathrm{~km} \mathrm{~s}^{-1}$. Brynildsen et al. (2003) also identified them as upwardly traveling acoustic waves.

O'Shea et al. (2002) measured time delays for the $3 \mathrm{~min}$ waves detected at different heights and estimated the propagating speeds for TRACE 1700 and O III $599.6 \AA$ to be in the range of $27-86 \mathrm{~km} \mathrm{~s}^{-1}$. They detected both upward and downward propagating waves. The result appreciably depends on the slit position (pixel number). This significantly complicates interpretation of the time delays detected for the waves at different levels. Marsh $\&$ Walsh (2006) showed that the 3 min oscillations in the transition zone above sunspots are directly connected to the waves 
in coronal loops. They supposed that global $p$-mode oscillations are involved in the process.

There are controversial opinions on plume- and fan-structure connection with the 3 min umbral oscillations. Brynildsen et al. (2004) showed that the 3 min umbral oscillations are limited to small coronal regions coinciding with bases of umbral coronal loops. They argue that these oscillations are not connected to plumes, whereas Jess et al. (2012) found a direct connection of the $3 \mathrm{~min}$ oscillations to coronal fans. They showed that the sources of the 3 min oscillations in coronal fans are seen to anchor into photospheric umbral dots with enhanced oscillation power. Analyzing the 3 min oscillations, Reznikova \& Shibasaki (2012) obtained contradictory results for two different sunspots. Wang et al. (2009) detected 12 and 25 min oscillations of intensity and line-of-sight (LOS) velocity in fan-like coronal structures above active regions in Hinode Extreme-Ultraviolet Imaging Spectrometer (EIS) data. They identified them as propagating slow magnetoacoustic waves.

The 3 min oscillations detected in the microwave range were found to replicate those recorded in the chromosphere of sunspots with a 50-s delay (Abramov-Maximov et al. 2011). This implies that the waves propagate from the chromosphere of an umbra to the upper levels.

Attempts to find a direct connection between photosphericchromospheric waves and coronal waves above sunspots have encountered difficulties. As a rule, instruments that obtain UV data to detect coronal waves do not observe in the $\mathrm{H} \alpha$ and He I $10830 \AA$ lines that are well suited for such a task. To perform a joint analysis, one has to use data from ground-based telescopes. The connection between waves in the corona and the deeper levels can be searched by direct comparison of the signal variations and by studying their spatial and temporal properties both visually and using cross-correlation.

This paper presents an attempt to make a joint analysis of the oscillations in a sunspot detected by ground-based observations and the Solar Dynamics Observatory (SDO) simultaneously. The data cover several levels of the solar atmosphere from the temperature minimum to the corona. Spatial power distribution of different oscillations is of interest, as well as their time lags. We tried to identify the frequencies for which the power spatial distribution better reproduces fan structures in the $171 \AA$ line.

The measurements of the time lag between the lowfrequency oscillations at the different heights were performed taking into account the oscillation propagation trajectory inclination. We hope that this allows us to make more accurate wave propagation speed calculations.

\section{Method and instruments}

Two types of data were used in the research. First, we observed the sunspot NOAA 11479 (Fig. 1) close to the disk center on 16 May 2012 for eight hours with a ground-based solar telescope at the Sayan Solar Observatory in two lines simultaneously: Si I $10827 \AA$ and He I $10830 \AA$. They represent the upper photosphere and the chromosphere, respectively. The coordinates of the spot were N15E15 at the beginning of the observation, which corresponds to the small angle between the line of sight and the normal to the Sun's surface. The analysis was performed at different frequency bands, $1-3.5 \mathrm{mHz}$ and $5-8 \mathrm{mHz}$.

The telescope resolution is usually about $1^{\prime \prime}$ owing to the Earth's atmosphere. The telescope photoelectric guide tracks the solar image with $1^{\prime \prime}$ accuracy for several hours of observations and compensates for image moving caused by the Sun's rotation.

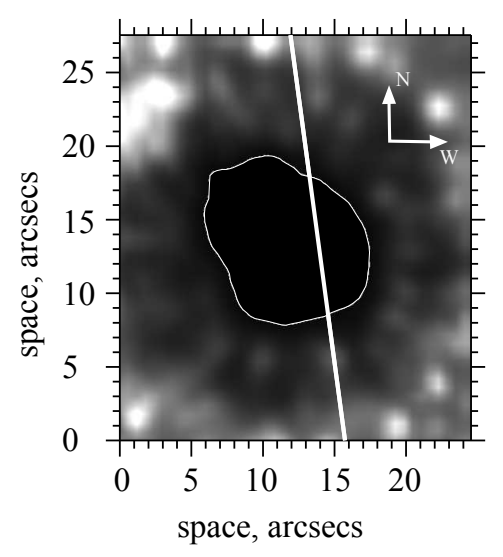

Fig. 1. Spot NOAA 11479 in the $1700 \AA$ continuum. The position of the slit relative to the spot is shown with the straight white line. The closed curve denotes the umbra boundary in the white light.

One camera sensor element corresponded to the $0.3^{\prime \prime}$ spatial resolution along the slit and $30 \mathrm{~m} \AA$ one along the spectrograph dispersion. The ultimate cadence was one second after the summation of every ten frames.

We used the Doppler-compensator method to calculate the line-of-sight velocity for the Si I-He I pair. The wavelength position shift corresponds to the measured line-of-sight velocity variation (Kobanov et al. 2009).

Also, we used for our analysis a series of Atmospheric Imaging Assembly (AIA) SDO data corresponding to the ground-based telescope observations. These observations comprise $6173 \AA, 1700 \AA, 304 \AA$, and $171 \AA$ spectral bands, which represent the lower photosphere, the upper photosphere, the transition region, and the corona, respectively (Reznikova et al. 2012).

To trace wave propagation from the photosphere to the chromosphere and the corona, we compared oscillations of the velocity and intensity signals filtered with Morlet wavelet. The time lags between the signals were determined by using the crosscorrelation method.

We based our analysis on the following premises: a) in sunspots the upwardly propagating waves travel along magnetic field lines; b) registered periodic variations of line intensities in the lower atmosphere are caused by temperature variations; c) we use the line formation heights accepted in the current literature for the spectral lines that we chose.

\section{Results}

Spatial localization of different frequency oscillations is in close relation to the magnetic field topology in sunspots. Both the early works (Sigwarth \& Mattig 1997; Kobanov \& Makarchik 2004; Tziotziou et al. 2006) and recent publications based on SDO data analysis (Reznikova \& Shibasaki 2012) note that lowfrequency oscillations concentrate in regions where the magnetic field lines are significantly inclined (i.e., in the penumbra), whereas the $5 \mathrm{mHz}$ and higher frequency oscillations are concentrated within the umbra boundaries.

First of all, we computed spatial distribution of the Fourier oscillation power for the $0.8 \mathrm{mHz}$ spectral bandpass centered at $3.3 \mathrm{mHz}$ (Fig. 2). Only the pixels with oscillation power exceeding $3 \sigma^{2}$ level are plotted on the power spectrum maps $(\mathrm{c}-\mathrm{e})$. The darker a point, the higher is the oscillation power in it within the specified band pass. The closed curves denote the umbra border. 


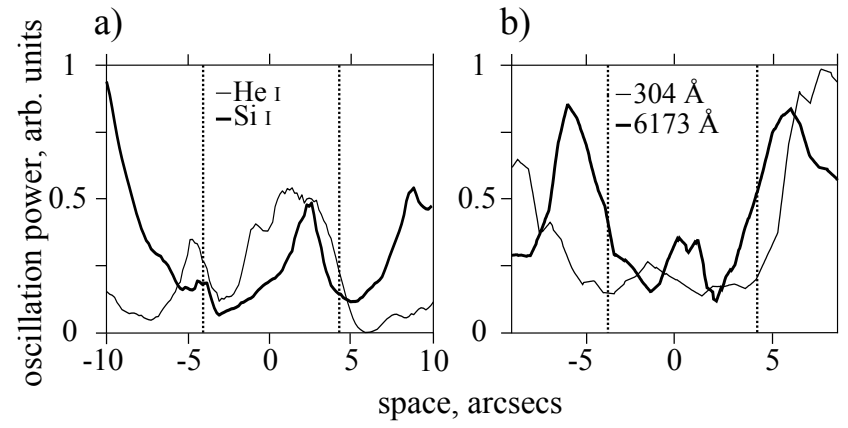

c)

d)

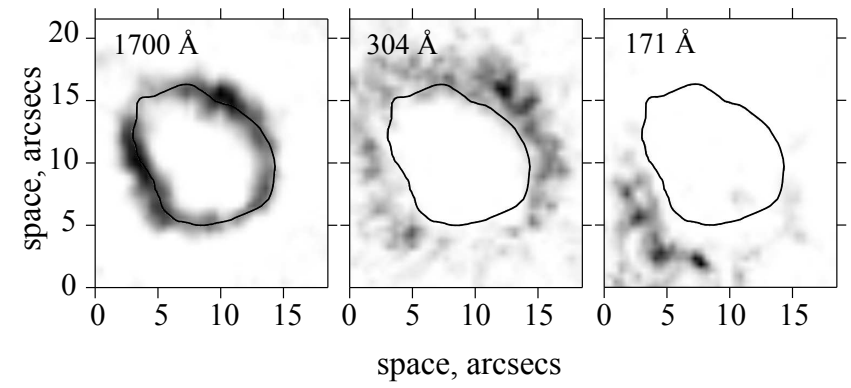

Fig. 2. Spatial distribution of the 5 min oscillation power a) along the slit of the ground-based telescope ( $\mathrm{Si}$ I $10827 \AA$ and $\mathrm{He}_{\text {I }} 10830 \AA$ ); b) along the slit in the $304 \AA$ and HMI Fe I $6173 \AA$ signals; c) in the $1700 \AA$ continuum; d) in the $304 \AA$ line; e) in the $171 \AA$ line. Only the pixels with oscillation power exceeding $3 \sigma^{2}$ level are plotted on the distributions on the panels $\mathbf{c}$ )-e). The darker a point, the higher is the oscillation power in it.

One can clearly see that the oscillation power is predominantly located in circle-shape structures; the areas of the circles increase with the line formation height. Proceeding from the assumption that these oscillations are manifestations of the magnetohydrodynamic waves propagating along the magnetic field lines, we attempted to measure the inclination of the lines by visually matching the oscillation power maps in different bands. To determine more accurate spatial correspondence, we analyzed sequences of the filtered signal wave trains in the vicinities of the supposed correspondence points and found correlation coefficients for them (Fig. 3a). Unambiguous correspondence was not found in every section along the umbra border, but in some points the cross-correlation coefficients reach 0.75 . The mean displacement $\Delta L$ of the circle structure boundaries is $2150 \mathrm{~km}$ (from 1800 to 3200 for different segments along the umbra boundary) for the $1700 \AA-304 \AA$ pair of lines. A minor discrepancy in positioning can make a contribution to the value spread for this pair of lines. The mean displacement of the circle boundaries for the $304 \AA-171 \AA$ pair of lines is about $1700 \mathrm{~km}$. The propagation trajectory inclination angle $\alpha$ was approximately estimated using a formula $\alpha=\arctan (\Delta L / \Delta z)$, where $\Delta z$ is the formation level difference. To calculate phase velocities, the propagation trajectory length $\mathrm{S}$ is roughly derived from a formula $S=\Delta L / \sin \alpha$. According to our preliminary results, which were calculated taking into account the differences in line formation heights, the average inclination of the magnetic field lines is 50-55 and 55-60 degrees for the height ranges corresponding to the $1700 \AA-304 \AA$ and $304 \AA-171 \AA$ pairs of lines, respectively (Fig. 4). Our estimations for the magnetic field line inclination at the umbra boundary are in compliance with the recent results from Jess et al. (2012) and Reznikova \& Shibasaki (2012).

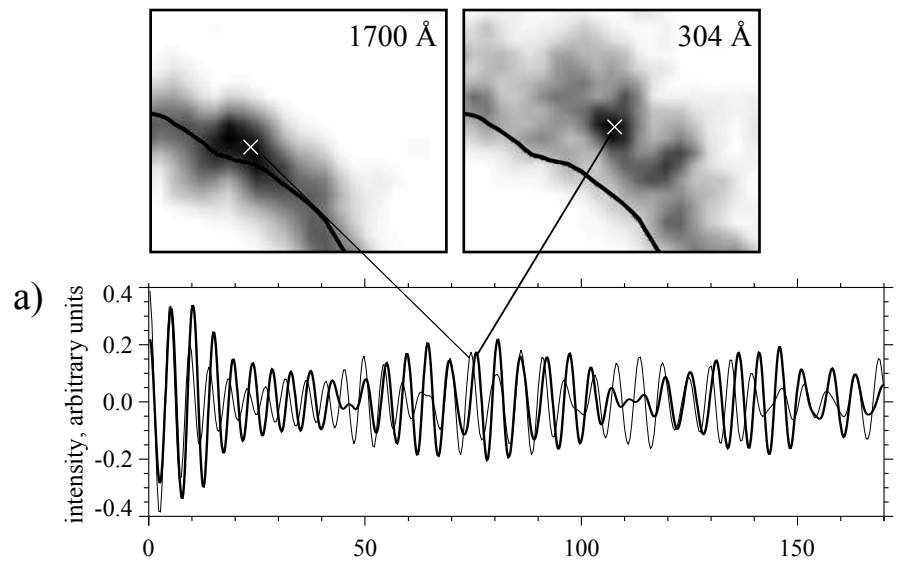

b)

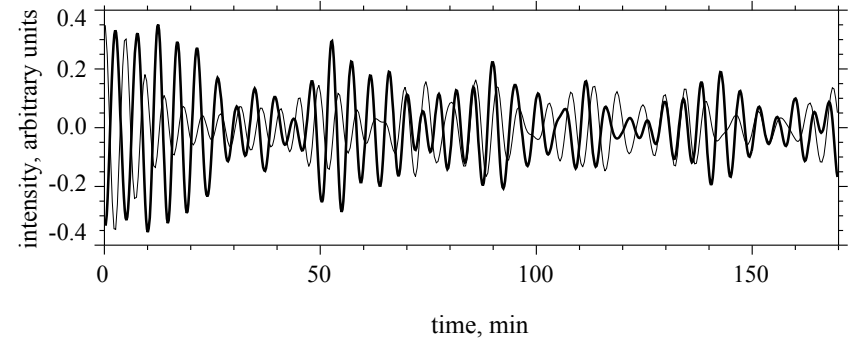

Fig. 3. Phase relations between the 5 min oscillations at $1700 \AA$ (thin) and $304 \AA$ (thick) levels. The signals were filtered in the $0.8 \mathrm{mHz}$ band centered at $3.3 \mathrm{mHz}$. a) The points for the different layers were selected accounting for the trajectory inclination, so that the filtered oscillation wave trains in the points showed the maximal correspondence (correlation coefficient is 0.7 ). b) The signals from the points located above each other at the line of sight at the point marked in the $1700 \AA$ panel show less correspondence (correlation coefficient is 0.35 ).

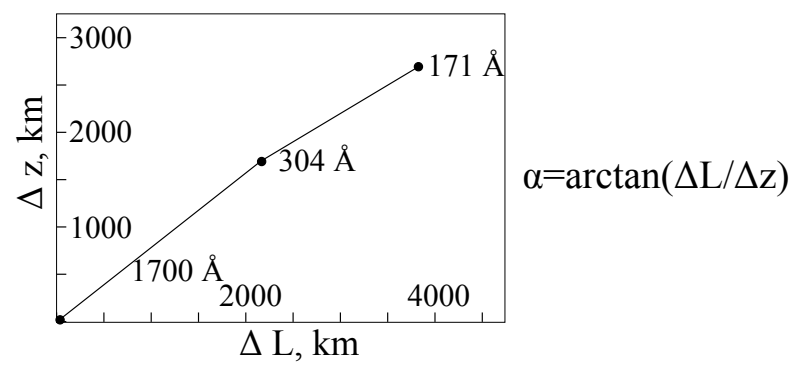

Fig. 4. Graphical interpretation of the 5 min wave trajectory. The broken line represents the average magnetic field inclination to the vertical derived from the horizontal displacements $(\Delta L)$ and the estimations of the differences between the line formation heights $(\Delta z)$.

Direct measurements of the signal phase difference at the two height levels are hindered owing to such an extreme inclination. Wave-train structures obtained at the line of sight for the two levels do not correspond, and an unambiguous determination of the phase lag turns out to be impossible (Fig. 3b). However, if we compare the signals in the points chosen with the account for the trajectory inclination, the similarity of the wave-train structures increases significantly (Fig. 3a). Nevertheless, different wave trains show different phase lags. This can be caused by the uncertainties of spatial alignment.

The inclination angle is harder to estimate from the $\mathrm{Si}$ I-He I pair because we are dealing with a one-dimensional cut of the spot by the spectrograph entrance slit; thus we can trace only 


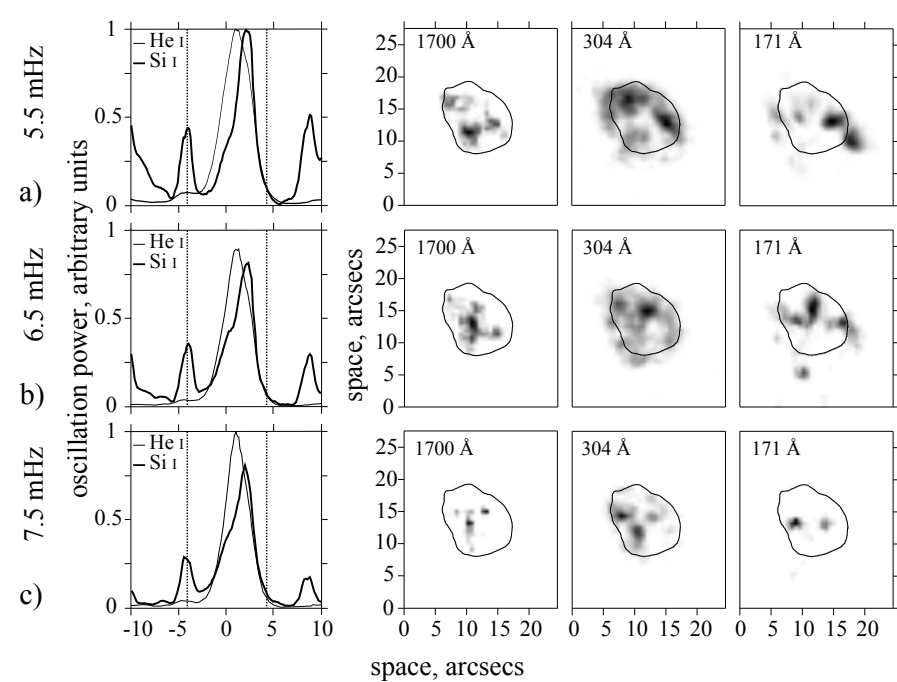

Fig. 5. Oscillation power spatial distribution for the bands $0.8 \mathrm{mHz}$ centered at a) $5.5 \mathrm{mHz}$; b) $6.5 \mathrm{mHz}$; c) $7.5 \mathrm{mHz}$. The first column presents the power distribution along the entrance slit for the $\mathrm{Si}$ I and $\mathrm{He}$ I lines.

displacements along the slit. The wave-train correspondence between the two pairs of points that we had is poor.

The distributions of $\mathrm{Si}$ I and He I 5 min oscillation power along the slit within the umbra are peaks with maxima located close to the umbra center and minima at the umbra borders (Fig. 2a). The He I peak is visibly broader than the Si I peak. The full width at half maximum of the Si I peak is 1.6 times smaller than that of the He I peak.

The 5 min oscillations in the $1700 \AA$ and $304 \AA$ lines are registered within the umbra, although it is not seen from the greyscale figures because the oscillation power in the penumbra is much higher than that in the umbra. The cuts along the slit of the Fe I $6173 \AA$ (HMI) and He II $304 \AA 5$ min oscillation power maps (Fig. 2) show the presence of peaks in the umbra, similar to those seen in the ground-based telescope observations. The displacement between the different layer umbral peaks is close to that observed for the $\mathrm{Si}$ I-He I pair.

The full width at the peak half maximum of the oscillation power distribution in the $\mathrm{He}_{\mathrm{I}}$ line exceeds that of the $\mathrm{Si}$ I line in the other frequency bands $(5-8 \mathrm{mHz})$ as well (Fig. 5). The ratio is 1.6 for the $5.5 \mathrm{mHz}$ and decreases to 1 with frequency increasing to $7.5 \mathrm{mHz}$. There is a $2^{\prime \prime}$ shift between the Si I and He I peaks in the oscillation power distributions (Fig. 5, first column). The positional angle does not cause such a displacement because the spot was observed close to the disk center. In our opinion, the displacement is caused by the magnetic field inclination.

Spatial power distributions for the lower frequency oscillations are presented in Fig. 6. As one can see, the $15 \mathrm{~min}$ oscillations are concentrated in the outer penumbra in the higher photosphere (1700 $\AA$ ), forming a circle structure. This structure expands in the transition zone ( $304 \AA$ ). Slender elements stretching radially become evident at this level. In the end, the $15 \mathrm{~min}$ oscillation power distribution in the $171 \AA$ line partially reproduces the fan picture seen in the corona. This fact gives evidence that the low-frequency oscillations of the $10-15$ min periods propagate upward from the outer penumbra and reach the corona level. It is of interest that previously Verwichte et al. (2009) found transverse $11 \mathrm{~min}$ oscillations of the coronal loops above active regions, which they identified as a fast magnetoacoustic kink mode.

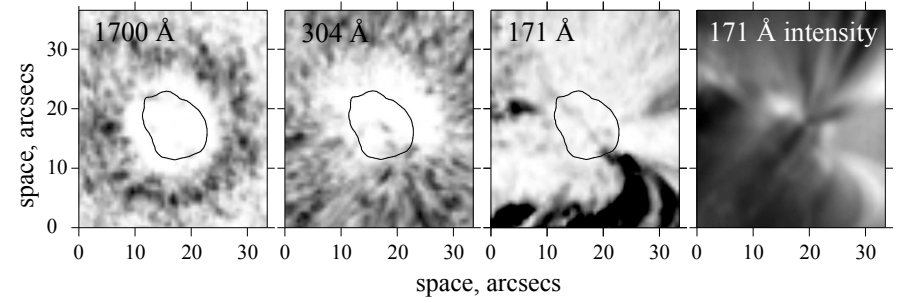

Fig. 6. Propagation of the low-frequency oscillations. First three panels from left to right: spatial distribution of the $15 \mathrm{~min}$ oscillation power in the $1700 \AA, 304 \AA$, and $171 \AA$. The fourth panel shows an image of the region in the $171 \AA$ line intensity. a)

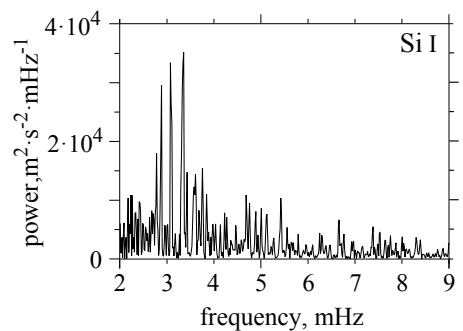

b)

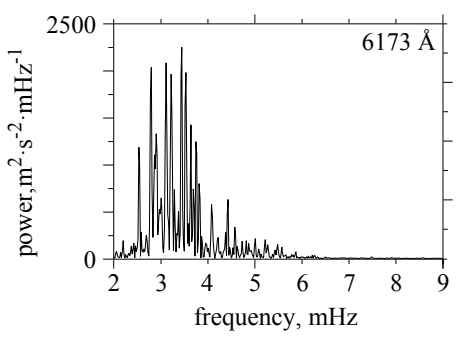

c)

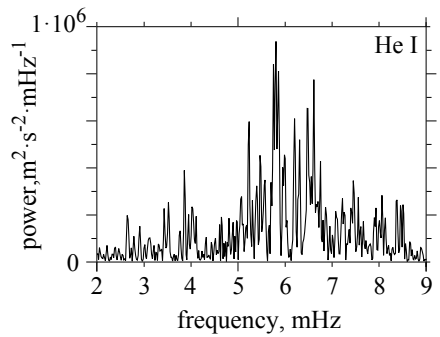

d)

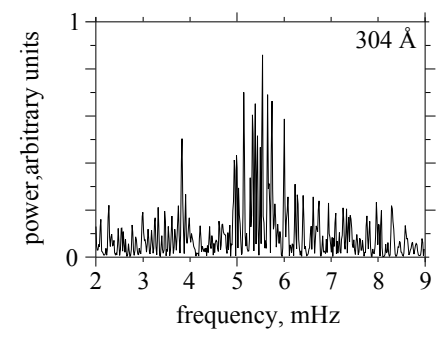

Fig. 7. Umbral oscillation power spectra from the photosphere to the transition zone for the a) Si I $10827 \AA$ LOS velocity; b) Fe I $6173 \AA$ LOS velocity; c) He I $10830 \AA$ LOS velocity; d) He II $304 \AA$ intensity signal.

Spatial distribution of the high-frequency oscillations $(5.5-7.5 \mathrm{mHz})$ differs from the distribution of the low-frequency oscillations (Fig. 5). First, the main power is concentrated within the umbra at the upper photospheric and chromospheric levels. Second, although the oscillation region expands significantly at the $304 \AA$ (transition region) and the $171 \AA$ (lower corona) heights, no circle structure appears, but fragmentariness becomes more apparent. Third, the number of umbral fragments showing presence of the oscillations decreases with increasing frequency. This means that the high-frequency oscillations concentrate in special points of the umbra. According to the finding of Jess et al. (2012), these points are the chromospheric umbral dots with the increased oscillation power.

The power spectra (Fig. 7) calculated in the different lines for the region that is cut by the slit in the sunspot umbra show that the main changes occur at the heights from the temperature minimum $(300-500 \mathrm{~km})$ to the transition region $(2000-2500 \mathrm{~km})$. Five-minute oscillations dominate explicitly in the Fe I $6173 \AA$ $(250 \mathrm{~km})$ and Si I $10827 \AA(540 \mathrm{~km})$ LOS velocity power spectra, whereas frequencies from 5.2 to $7 \mathrm{mHz}$ dominate at the He I $10830(2100 \mathrm{~km})$ and $304(2300 \mathrm{~km})$ line formation heights. This indicates that the $3 \mathrm{~min}$ umbral oscillations evolve and sharply amplify directly in a chromosphere cavity 

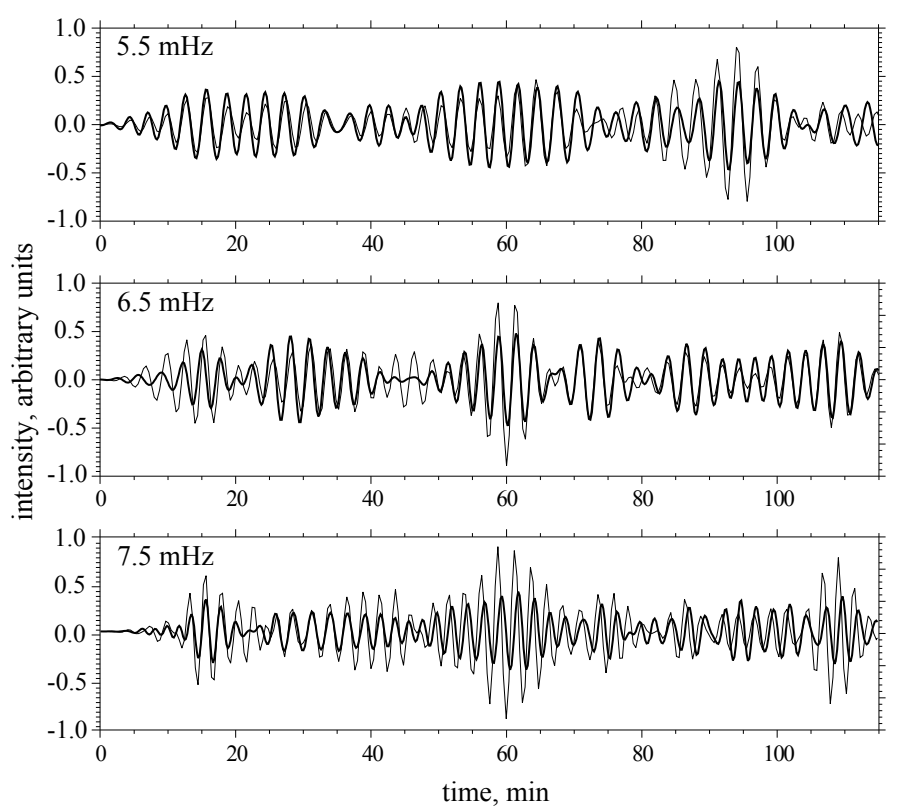

Fig. 8. Signals of the $1700 \AA$ (thin) and $304 \AA$ (thick) lines filtered in $0.8 \mathrm{mHz}$ band centered at $5.5 \mathrm{mHz}, 6.5 \mathrm{mHz}$, and $7.5 \mathrm{mHz}$. The $304 \AA$ signal was shifted backwards by $\delta t$, so that the correlation coefficients between the signals were maximal ( $\delta t$ is $36 \mathrm{~s}, 60 \mathrm{~s}$, and $52 \mathrm{~s}$ for the $u p$ per, middle, and bottom panel, respectively). The $1700 \AA$ signal was amplified by a factor of $3.3,5$, and 6.7 for the first, second, and third band, respectively.

(Zhugzhda et al. 1985; Botha et al. 2011). We obtained similar results earlier from $\mathrm{H} \alpha$ and $\mathrm{Fe}_{\mathrm{I}} 6569 \AA$ observations (Kobanov et al. 2011).

Taking into account the horizontal shift of the oscillation between the layers, we measured time lags for the $5 \mathrm{~min}$ oscillations. For the $1700 \AA-304 \AA$ pair of lines, the value of the time lag shows a great spread from $-20 \mathrm{~s}$ to $108 \mathrm{~s}$, where negative values signify the photospheric signal lagging behind the chromospheric one. A significant spread can appear even in one spatial point at different time intervals. Reznikova et al. (2012) note the similar feature of time lag measurements between the oscillations above sunspots. The average lag between the $\mathrm{Si}$ I and $\mathrm{He}$ I signals is $47 \mathrm{~s}$; it shows a great spread, too. The $304 \AA-171 \AA$ pair shows the time lags from -12 to 48 s, with the average value being $24 \mathrm{~s}$. Poor correspondence between the signal wave trains in the layers does not mean that there are no waves propagating through the layers upward. This may signify the presence of both standing and traveling waves in the volume researched. The presence of standing waves in an object does not exclude the possibility of this object being a source of waves propagating in the surrounding medium. If the resonator boundary does not reflect the wave thoroughly, this makes it an oscillation source. The majority of oscillation sources contain resonators. The ratio between these two types of waves influences the results obtained. (see, e.g., Kobanov et al. 2011). Also, this may signify that the magnetic field loops presenting in the lower layer either do not reach the upper level returning to the photosphere or divert from the line of sight significantly.

The time lag varies from 36 to $84 \mathrm{~s}$ and reaches 0 at some spatial points for the $1700 \AA-304 \AA$ pair in the $0.8 \mathrm{mHz}$ band passes centered at 5.5, 6.5, 7.5 $\mathrm{mHz}$ (see Fig. 8). We chose the points located above each other at the line of sight for our analysis. Because the high-frequency oscillations propagate along the

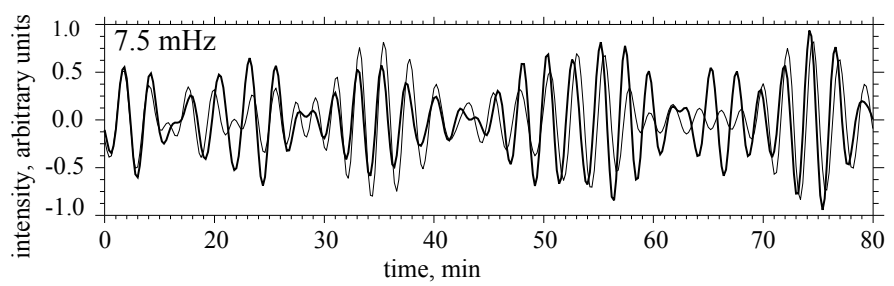

Fig. 9. Unshifted signals of the $304 \AA$ (thick) and $171 \AA$ (thin) lines filtered in $0.8 \mathrm{mHz}$ band centered at $7.5 \mathrm{mHz}$. The $171 \AA$ signal was amplified by a factor of 2.3 .

vertical and close-to-vertical magnetic field lines in the central umbra, the visibility of the traveling waves is hardly influenced by a projection effect. The wave-train structure resemblance indicates that the points located at the oscillation propagation trajectory are used. Besides, wave-train visual analysis helps avoid the $2 \pi$ uncertainty, which occurs when one measures phase lags. In the $304 \AA-171 \AA$ pair, the lag varies from 12 to 24 s (see, e.g., Fig. 9). One can see that the wave trains in Figs. 8 and 9 correspond to each other much better than those of the lower frequencies. This is because the higher frequencies "prefer" to propagate along the vertical magnetic flux.

Taking the height difference of $1700 \mathrm{~km}$ for the $1700 \AA-304 \AA$ pair and $1000 \mathrm{~km}$ for the $304 \AA-171 \AA$ pair (Reznikova et al. 2012) and bearing in mind the diversion from the vertical propagation, we found the average phase velocities to be $24 \mathrm{~km} \mathrm{~s}^{-1}$ and $42 \mathrm{~km} \mathrm{~s}^{-1}$ for the first and the second pair, respectively, for the frequency band centered at $3.3 \mathrm{mHz}$. For the frequencies from 5 to $8 \mathrm{mHz}$, the phase velocities are $28 \mathrm{~km} \mathrm{~s}^{-1}$ and $55 \mathrm{~km} \mathrm{~s}^{-1}$, respectively. The uncertainties of these calculations are relatively great (from $20 \%$ to $60 \%$ ) owing to the inconsistencies in the determined time lags.

It is of interest to note that for all the frequencies analyzed, the $304 \AA$ line intensity oscillation amplitude exceeds appreciably those of the other lines, both at the higher and lower levels (see also Reznikova et al. 2012).

We found that the phase difference between the intensity and velocity signals (I-V) for the Fe I $6173 \AA$ and Si I $10827 \AA$ lines is close to $90^{\circ}$ on average (Fig. 10), which is characteristic for acoustic waves under the adiabatic approximation. This difference is ambiguous and close to $180^{\circ}$ for the upper chromosphere He $10830 \AA$ line at individual time intervals. The situation with the He I $10830 \AA$ line is complicated by the fact that its profile is largely influenced by the UV radiation coming from the upper layers of the solar atmosphere. We assume that the observed waves are slow magnetoacoustic waves propagating upward along the magnetic field lines.

We believe that intensity-temperature phase relationship research in the corona is also important. To this end, we intend to implement the promising method described in Aschwanden \& Boerner (2011) and Aschwanden et al. (2013) in our future work.

\section{Conclusions}

The spatial distribution of 5 min oscillation power looks like a circle-shape structure matching the umbra border at the photospheric level. The circle expands, and its boundaries move away from the inner penumbra border at the higher levels (304 $\AA$ and $171 \AA$ ). This indicates that $5 \mathrm{~min}$ oscillations propagate along appreciably inclined magnetic tubes in the spot. The inclination 

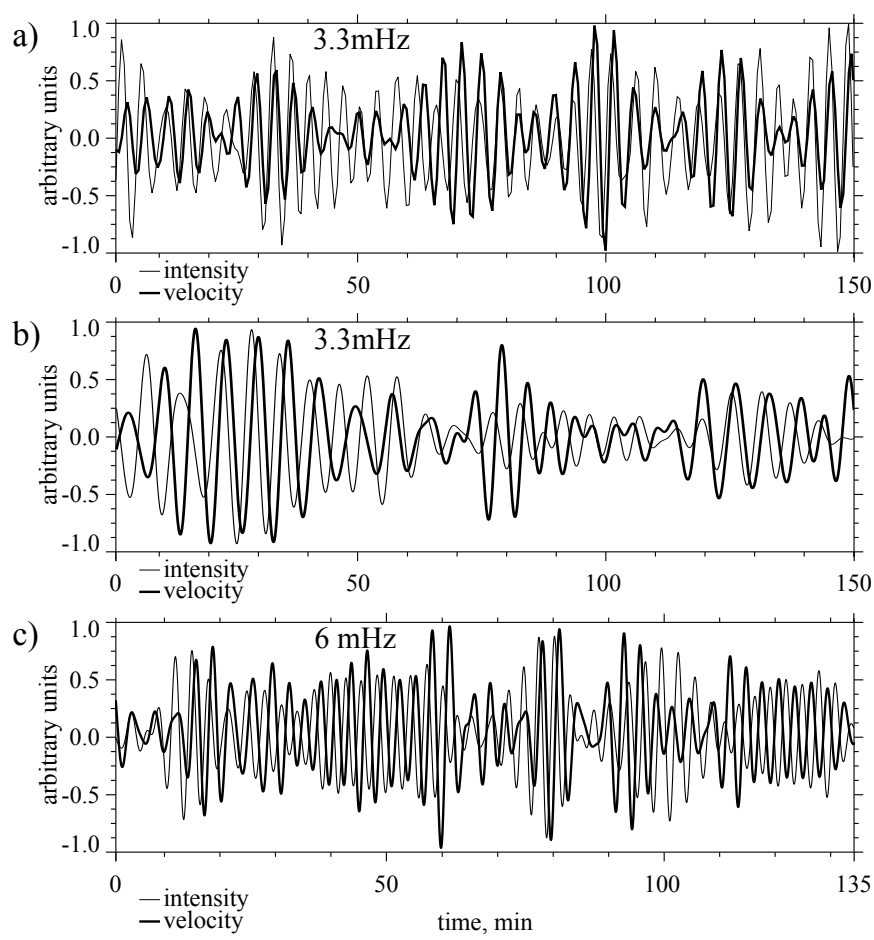

Fig. 10. Phase relationships between the intensity (thin) and velocity (thick) signals for the a) $\mathrm{Fe}_{\mathrm{I}} 6173 \AA$ line; b) Si $10827 \AA$ line; c) He I $10830 \AA$ line.

of the tubes reaches 50-60 degrees at the levels of the transition region and the lower corona.

While the 10-15 min oscillation power distribution shows a circle structure at the lower layers as well, it clearly resembles fan structures at the coronal level (it is of interest to notice that the coronal-level 5 min distribution does not show such a resemblance, at least up to the $171 \AA$ formation level). As is known, 8-15 min oscillations dominate in the photospheric and chromospheric outer penumbra (see Sigwarth \& Mattig 1997; Kobanov 2000). This indicates that these oscillations come to the corona from the lower layers of the solar atmosphere.

The main oscillation power in the $5-8 \mathrm{mHz}$ range is concentrated within the umbra boundaries both at the temperatureminimum and chromospheric levels. The oscillation region covers the greater area at the transition region and at lower corona levels than that at the photospheric level. The higher frequency oscillations are concentrated in small regions inside the umbra, probably coinciding with the loop bases anchored in umbral dots.

We found no evidence of any connection between the 3 min oscillations and fan structures, at least for the $171 \AA$ formation level. In this respect our results agree with those of Brynildsen et al. (2004). It is reasonable to suppose that the connection between the 3 min oscillations and fan structures is more evident in the higher coronal levels.

We deduced that the average phase velocities are $26 \pm 15 \mathrm{~km} \mathrm{~s}^{-1}$ and $50 \pm 10 \mathrm{~km} \mathrm{~s}^{-1}$ in the frequency range 3-8 mHz for the $1700 \AA-304 \AA$ and $304 \AA-171 \AA$ height levels, respectively.
To determine the phase lags of the waves between the height levels more correctly, it is necessary to take into account the inclination of the magnetic field lines, i.e., to analyze the points not taken at the line of sight but rather chosen considering the displacement in the horizontal plane. As a result, the direct phaselag detection procedure becomes considerably more complicated. This especially applies to the low-frequency oscillations.

Acknowledgements. This study was supported in part by the RFBR research project No. 12-02-33110 mol_a_ved and the Grant of the President of the Russian Federation No. MK-497.2012.2, Russian Federation Ministry of Education and Science state contract No. 14.518.11.7047 and agreement No. 8407. We are grateful to the NASA/SDO science team for providing the data. We acknowledge Y. M. Kaplunenko for his help in preparing the English version of the paper.

\section{References}

Abramov-Maximov, V. E., Gelfreikh, G. B., Kobanov, N. I., Shibasaki, K., \& Chupin, S. A. 2011, Sol. Phys., 270, 175

Aschwanden, M. J., \& Boerner, P. 2011, ApJ, 732, 81

Aschwanden, M. J., Fletcher, L., Schrijver, C. J., \& Alexander, D. 1999, ApJ, 520,880

Aschwanden, M. J., Boerner, P., Schrijver, C. J., \& Malanushenko, A. 2013, Sol. Phys., 283, 5

Balthasar, H., \& Wiehr, E. 1990, A\&A, 237, 243

Beckers, J. M., \& Tallant, P. E. 1969, Sol. Phys., 7, 351

Bloomfield, D. S., Lagg, A., \& Solanki, S. K. 2007, ApJ, 671, 1005

Bogdan, T. J., \& Judge, P. G. 2006, Roy. Soc. London Phil. Trans. Ser. A, 364, 313

Botha, G. J. J., Arber, T. D., Nakariakov, V. M., \& Zhugzhda, Y. D. 2011, ApJ, 728,84

Brynildsen, N., Maltby, P., Brekke, P., Redvik, T., \& Kjeldseth-Moe, O. 2003, Adv. Space Res., 32, 1097

Brynildsen, N., Maltby, P., Foley, C. R., Fredvik, T., \& Kjeldseth-Moe, O. 2004, Sol. Phys., 221, 237

Centeno, R., Collados, M., \& Trujillo Bueno, J. 2009, ApJ, 692, 1211

De Moortel, I., Ireland, J., Hood, A. W., \& Walsh, R. W. 2002, A\&A, 387, L13

Giovanelli, R. G. 1972, Sol. Phys., 27, 71

Jess, D. B., De Moortel, I., Mathioudakis, M., et al. 2012, ApJ, 757, 160

Kobanov, N. I. 2000, Sol. Phys., 196, 129

Kobanov, N. I., \& Makarchik, D. V. 2004, A\&A, 424, 671

Kobanov, N. I., Kolobov, D. Y., \& Makarchik, D. V. 2006, Sol. Phys., 238, 231

Kobanov, N. I., Kolobov, D. Y., Sklyar, A. A., Chupin, S. A., \& Pulyaev, V. A. 2009, Astron. Rep., 53, 957

Kobanov, N. I., Kolobov, D. Y., Chupin, S. A., \& Nakariakov, V. M. 2011, A\&A, 525, A41

Lites, B. W. 1992, in NATO ASIC Proc. 375: Sunspots. Theory and Observations, eds. J. H. Thomas, \& N. O. Weiss, 261

Marsh, M. S., \& Walsh, R. W. 2006, ApJ, 643, 540

Nakariakov, V. M., Ofman, L., Deluca, E. E., Roberts, B., \& Davila, J. M. 1999, Science, 285, 862

O’Shea, E., Muglach, K., \& Fleck, B. 2002, A\&A, 387, 642

Reznikova, V. E., \& Shibasaki, K. 2012, ApJ, 756, 35

Reznikova, V. E., Shibasaki, K., Sych, R. A., \& Nakariakov, V. M. 2012, ApJ, 746,119

Rouppe van der Voort, L. H. M., Rutten, R. J., Sütterlin, P., Sloover, P. J., \& Krijger, J. M. 2003, A\&A, 403, 277

Sigwarth, M., \& Mattig, W. 1997, A\&A, 324, 743

Thomas, J. H., \& Weiss, N. O. 2008, Sunspots and Starspots (Cambridge University Press)

Tsiropoula, G., Alissandrakis, C. E., \& Mein, P. 2000, A\&A, 355, 375

Tziotziou, K., Tsiropoula, G., Mein, N., \& Mein, P. 2006, A\&A, 456, 689

Verwichte, E., Aschwanden, M. J., Van Doorsselaere, T., Foullon, C., \& Nakariakov, V. M. 2009, ApJ, 698, 397

Wang, T. J., Ofman, L., Davila, J. M., \& Mariska, J. T. 2009, A\&A, 503, L25

Zhugzhda, I. D., Locans, V., \& Staude, J. 1985, A\&A, 143, 201

Zirin, H., \& Stein, A. 1972, ApJ, 178, L85 